\title{
Reactivation of Epstein-Barr virus in Sjögren's syndrome
}

\author{
Robert I. Fox, Mario Luppi*, Ho-Il Kang, and Pavel Pisa** \\ Department of Rheumatology and Immunology, Scripps Clinic and Research Foundation, \\ 10666 North Torrey Pines Road, La Jolla, CA 92037, USA
}

Summary. Sjögren's syndrome (SS) is a chronic autoimmune disease characterized by severe dryness of the eyes and mouth, resulting from lymphocytic infiltration of the lacrimal and salivary glands. SS may exist as a primary condition (primary SS, $\left.1^{\circ} \mathrm{SS}\right)$ or as a secondary condition $\left(2^{\circ} \mathrm{SS}\right)$ in association with rheumatoid arthritis, systemic lupus erythematosus, or progressive systemic sclerosis. In some $1^{\circ} \mathrm{SS}$ patients, there may be involvement of the extraglandular organs, including skin, kidney, liver, lung and nervous system. Furthermore, these patients may develop a lymphoproliferative syndrome that includes lymphadenopathy and increased risk of lymphoma. In the pathogenesis of SS, a role for Epstein-Barr virus (EBV) has been suggested because: (a) EBV is present in salivary gland epithelial cells of normal individuals and exaggerated immune responses against EBV could play a role in the destruction of salivary glands in SS; (b) SS salivary gland biopsies contain increased levels of EBV DNA in comparison to normal salivary glands, indicating viral reactivation and inability of lymphoid infiltrates to control EBV replication in SS patients; and (c) salivary gland epithelial cells in SS patients express high levels of HLA-DR antigens and may present EBV-associated antigens to immune T cells in SS patients. Therefore, SS may represent a situation in which genetically predisposed individuals (i. e., HLA-DR3-DQA4-DQB2) have a persistent but ineffectual T cell immune response against EBV at its site of latency. Among 14 non-Hodgkin's lymphomas that developed in SS patients, EBV DNA was detected in increased amounts in the tumor tissue of one patient. Characterization of this tumor DNA revealed: (a) polyclonal immunoglobulin gene rearrangements; (b) EBV DNA with an unusual restriction fragment length polymorphism pattern involving the Bam M fragment; and (c) EBV terminal repeat sequences suggestive of viral replication, similar to those reported in EBV lymphomas occurring in other immunocompromised individuals. Early recognition of this clinical problem may allow beneficial use of antiviral agents.

\footnotetext{
* Present address: Department of Hematology, University of Modena, Modena, Italy ** Present address: Department of Immunology, Karolinska Institutet, P.O. Box 60400, S-10401 Stockholm, Sweden Offprint requests to: R. I. Fox
} 


\section{Background}

In 1888, Johann van Mikulicz Radecki described a 42-year-old Prussian farmer with enlargement of lacrimal and salivary glands "consisting of small round cells", [28]. In 1933, Henrik Sjögren described the association of keratoconjunctivitis sicca (KCS), dry mouth (xerostomia) and rheumatoid arthritis (RA) [37]. Although this association of symptoms is generally known as Sjögren's syndrome, it is occasionally referred to as "Gougerout-Sjögren's" syndrome to acknowledge the earlier report by Gougerout in 1925 [20]. In 1953, Morgan and Castleman demonstrated that a histologically similar infiltrate was present in both SS and Mickulicz disease [29]. Bloch et al. [4] suggested that SS should be subdivided into primary SS $\left(1^{\circ} \mathrm{SS}\right)$ and secondary SS $\left(2^{\circ} \mathrm{SS}\right) .2^{\circ} \mathrm{SS}$ was defined as sicca symptoms in association with particular diseases such as RA, systemic lupus erythematosus (SLE), progressive systemic sclerosis (PSS) or dermatomyositis, whereas $1^{\circ}$ SS patients lacked those particular disease associations.

\section{Criteria for diagnosis and epidemiology}

The specific criteria for diagnosis of SS have been an issue of considerable debate; those used at our institution are listed in Table 1. In particular, we require objective evidence of dryness, lymphocytic infiltrates and autoantibodies. This is shown schematically in Fig. 1, in which the SS patient has enlarged parotid glands and dry eyes, manifest by the decreased wetting of paper strips placed under the lower eyelids (the Schirmer's test). There are many different causes of dryness, ranging from drug side effects to the normal process of aging. Therefore, any studies that search for common epidemiological features of SS must use stringent criteria to avoid patients with other causes of their sicca symptoms.

$1^{\circ}$ SS has a world-wide distribution and probably has a frequency about 1/3000. In the United States, SS occurs in the Caucasoid, Negroid and Mexican populations. Among 200 consecutive $1^{\circ}$ SS patients evaluated at Scripps Clinic, 180 were females with median age 54 years at time of onset. In 10 patients, age of onset was 12 years or younger. A positive family history of systemic autoimmune disease involving parent (mother) or sister was confirmed in 14 families. $1^{\circ} \mathrm{SS}$ in a sibling was found in only $2 / 14$ families, and SLE in 10/14 families. Thus, an increased risk towards autoimmune disease may exist in families with a $1^{\circ}$ SS patient. However, the disease most commonly detected in $1^{\circ} \mathrm{SS}$ families was SLE. This suggests that a similar genetic factor[s] predisposes to both $1^{\circ}$ SS and SLE. An additional factor such as environmental agents may lead to the development of a specific disease such as SLE or $1^{\circ}$ SS.

\section{Autoantibodies and autoantigens in SS}

The most common autoantibodies in $1^{\circ} \mathrm{SS}$ patients are antinuclear antibodies (ANA), that result from reactivity against the SS-A and SS-B antigens. Initial 
Table 1. Criteria for diagnosis of primary and secondary Sjögren's syndrome (SS)

I. Primary SS

A. Symptoms and objective signs of ocular dryness

1. Schirmer test $<8-\mathrm{mm}$ wetting per 5 min

2. Positive rose bengal or fluorescein staining of cornea and conjunctiva to demonstrate keratoconjunctivits sicca

B. Symptoms and objective signs of dry mouth

1. Decreased parotid flow rate using Lashley cups or other methods

2. Abnormal biopsy of minor salivary gland (focus score of $\geq 2$ based on average of 4 evaluable lobules)

C. Evidence of a systemic autoimmune disorder

1. Elevated rheumatoid factor $\geq 1: 320$

2. Elevated anti-nuclear antibody $\geq 1: 320$

3. Presence of anti-SS-A [Ra] or anit-SS-B [La] antibodies

II. Secondary SS

Characteristic signs and symptoms of SS (described above) plus clinical features sufficient to allow a diagnosis of RA, SLE, polymyositis or scleroderma

III. Exclusions: sarcoidosis, pre-existent lymphoma, acquired immunodeficiency disease and other known causes of keratitis sicca or salivary gland enlargement

RA, Rheumatoid arthritis; SLE, systemic lupus erythrematosus

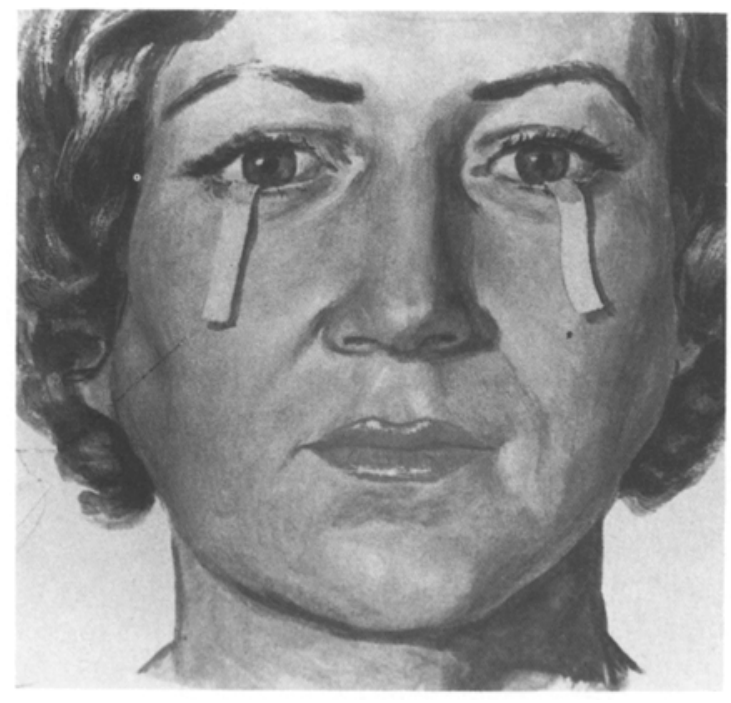

Fig. 1. Schematic illustration of a patient with Sjogren's syndrome (SS). The swollen parotid glands are present and dry eyes are indicated by the absence of tear flow onto paper strips inserted under the lower eyelids (the Schirmer's test)

studies suggested that SS-A (also known as "Ro") antigen was a single 60-kDa protein; subsequent reports demonstrated proteins reactive with anti-SS-A antibody including a 52-kDa protein in all nucleated cells [3] and a 54-kDa protein 
in non-nucleated red blood cells $[21,22,31]$. Anti-SS-A antibody also is found in low titers in normal adults [18] and ina subset of patients with multiple myeloma [36].

Lerner et al. [24] demonstrated the association of SS-A protein with a novel class of small RNA designated hY1-hY5 (h for human and Y for cytoplasmic) RNA, although there is now evidence that such RNA are also intranuclear. The hY RNA share many similarities including sequence, size (83-112 bp), secondary sequence and abundance (about $10^{5}$ copies/cell) [43]. It is not known whether all SS-A proteins bind to hY RNA, nor if they are structurally or functionally related. Each of the genes encoding SS-A proteins appears to be single copy and nonpolymorphic, so the reason that SS-A is a target for immune response remains unknown. It has been proposed that nascent EBV proteins are associated with the SS-A/SS-B complex and may serve to initiate autoimmune responses.

The SS-B [La] protein is part of the same ribonuclear particle that contains SS-A. Antibodies against SS-B are much more closely associated with clinical symptoms of SS and are present in about $40 \%-50 \%$ of these patients. Anti-SS-B antibodies almost invariably occur in the presence of anti-SS-A antibodies, although the converse is not true. The SS-B protein binds to the $3^{\prime}$ uridylate tail of RNA transcripts derived from RNA polymerase III, including U6 cellular RNA, adenovirus RNA (VA I and VA II), EBV RNA (EBER) and leader RNA of some negative-strand viruses such as vesicular stomatitis virus. Recent studies have shown that SS-B protein is essential in the biogenesis of these RNA. The fact that SS-B is associated with precursors of 5s and tRNA, but not with their corresponding mature species has suggested that SS-B may play a role in the maturation of these polymerase III transcripts. In addition, SS-B may bind to U1 RNA, an RNA polymerase II transcript.

\section{Histology}

The characteristic finding in SS is lymphocytic infiltration of the salivary and lacrimal gland (Fig. 2, frame A). The majority of salivary and lacrimal gland lobules are involved and infiltrates are more prominent in the central portion of the lobule than in the periphery. Well-defined germinal centers are infrequent in minor salivary gland biopsy specimens but occasionally present in parotid and submandibular infiltrates. Normal parenchymal structures such as acini and ducts may be extensively replaced by these infiltrates. "Activated" lymphocytes with large, vesicular nuclei and prominent nucleoli are frequently present and may be difficult to distinguish from the lymphocytes in lymphoma [7, 32]. At higher power, frame $\mathrm{C}$ shows some acini that are relatively intact but lymphocytes are present within the acinar structure (arrow). Frame D shows a duct with an increased number of infiltrating lymphocytes (arrows) and degenerating epithelial cells. Frames $\mathrm{E}$ and $\mathrm{F}$ demonstrate the high endothelial venules with red blood cells in the lumen and lymphocytes adherent to the vessel wall. Of importance, electron-dense immune complex deposits are not present at the basement membrane surrounding the blood vessel or epithelial cells. Taken together, the 


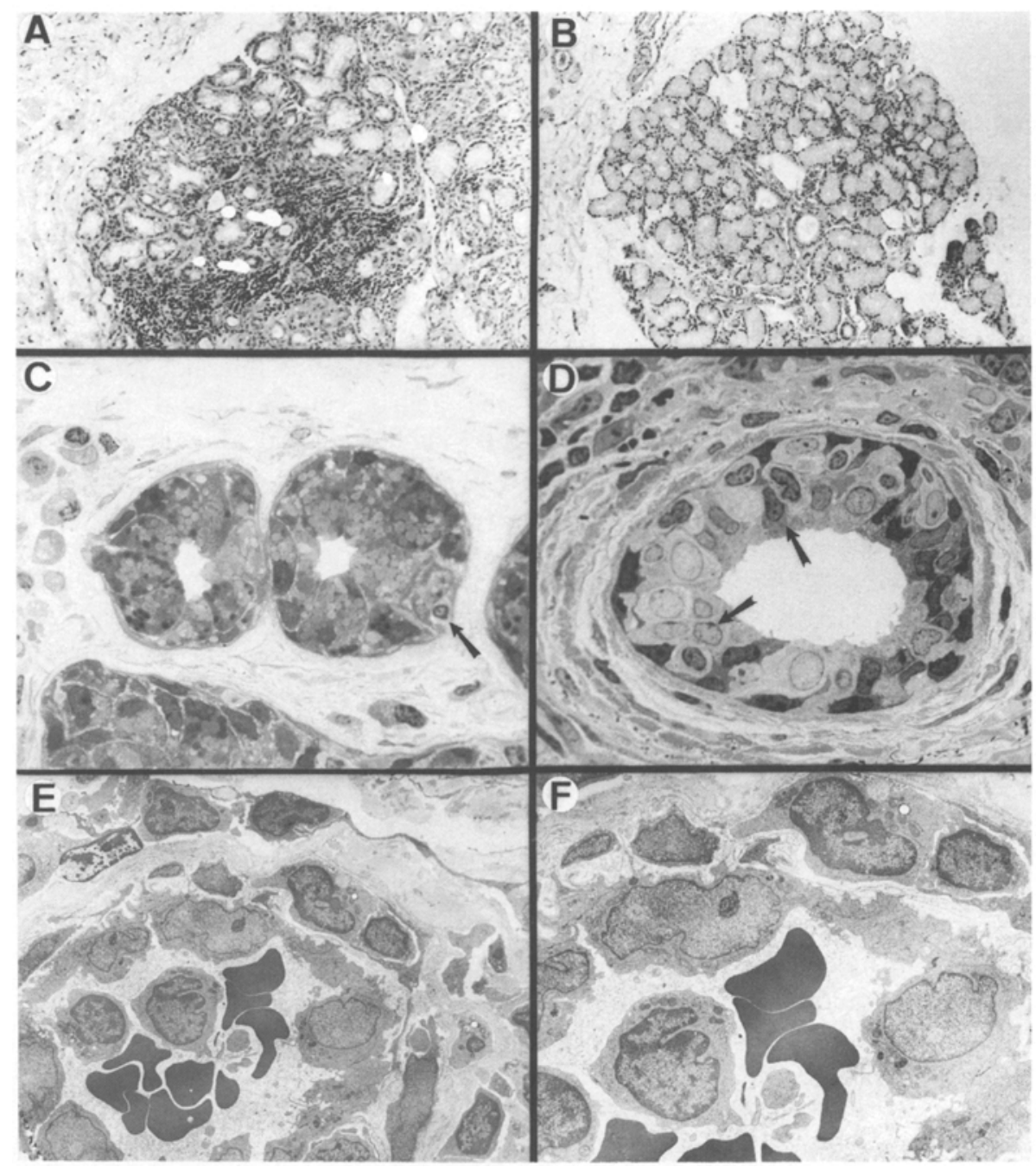

Fig. 2A-F. Salivary gland biopsies from a patient with SS and from a normal individual. A shows a biopsy from a SS patient, while $\mathbf{B}$ shows a normal salivary gland for comparison. In the SS biopsy, the initial lymphocytic infiltrate occurs in the central portion of the salivary gland. These infiltrates occur around a central blood vessel that undergoes a morphological change to become a high endothelial venule. Different stages of the inflammatory process within this SS gland are apparent with early lymphocytic infiltration (C) and extensive infiltration (D). Using immunohistological methods, these lymphocytes are shown to be $\mathrm{CD}^{+} \mathrm{T}$ cells. In $\mathbf{C}$ and $\mathrm{D}$, the lymphocytes are in direct contact with the salivary gland epithelial cells and beneath the basement membrane enclosing acini and ducts. High endothelial venules (containing red blood cells) and lymphocytes adherent to the vascular endothelium ean be seen $(\mathbf{E}$ and $\mathbf{F}$ ). Of importance, electron-dense immune deposits are not seen at the basement membrane around the blood vessels, nor at the glandular epithelial cells. These results support a cell mediated mechanism of damage, rather than an immune complex mediated model of pathogenesis 
light and elctron microscopic appearance of salivary gland infiltrates in SS suggests a cell-mediated destruction rather than an immune complex deposition mechanism.

Within the salivary glands, the acini produce either "serous" (i.e., watery secretions high in enzyme content) or "mucinous" (tenacious secretions of mucopolysaccharides) secretions. The salivary glands are rich sources of growth factors including epidermal growth factor, nerve growth factor, insulin-like growth factor and lactoferrin. In SS, the immunological destruction appears to be directly aimed at the "serous" glands and this partly accounts for the tenacious secretions that occur in the eyes and mouth of these patients. At the electron microscopic level, most SS salivary gland acinar cells have a decreased number of secretory granules, suggesting that their contents may be liberated as a result of the immune attack [6]. Nuclei of the acinar cells appeared relatively unremarkable except for prominent nucleoli. Tubuloreticular structures may be present and may represent a nonsepcific response to epithelial cell injury [6]. It is likely that the growth factors and cytokines released from the injured salivary gland cells play an important role in the pathogenesis of SS.

To characterize the cell-cell interactions in SS, immunohistological methods have been used to identify lymphocyte subsets [1]. The majority of lymphocytes are mature $\mathrm{T}$ cells $\left(\mathrm{CD}^{+}\right)$of the $\mathrm{T}$ helper $\left(\mathrm{CD}^{+}\right)$subset. Some $\mathrm{T}$ suppressor cells $\left(\mathrm{CD}^{+}\right)$also were present. Of particular interest, certain lymphocyte subsets were present in the salivary gland infiltrates but were absent from the blood. Conversely, other lymphocyte subsets (such as natural killer cells) were present in blood but were absent from the salivary gland infiltrates [10]. Similarly, the functional properties of $\mathrm{T}$ cells eluted from the salivary gland of SS patients, such as interleukin (IL) 2 production, differ from the properties of $\mathrm{T}$ cells in the peripheral blood of the same patient [11]. These results emphasize the need to study the target organ to elucidate pathogenesis, since alterations in lymphocytes occurring in blood may not be present in the target organ.

Salivary gland epithelial cells in biopsies from SS patients react with antiHLA-DR antibodies, in contrast to normal salivary gland biopsies that lack antiHLA-DR reactivity $[12,25]$. The induction of HLA-DR on the epithelial cells may play an important role in pathogenesis, since $\mathrm{CD}^{+} \mathrm{T}$ cells (the major lymphocyte subset in the SS salivary gland) only interact with peptide antigen presented by HLA-DR molecules. The induction of epithelial cell HLA-DR molecules is probably due to local production of interferon- $\gamma$ by $\mathrm{T}$ cells, since monoclonal antibody against this cytokine eliminates the HLA induction [12].

\section{EBV infection of normal individuals}

Initial EBV infection is controlled by "natural killer" cells and long-term protection is mediated by $\mathrm{T}$ lymphocytes of the $\mathrm{CD} 8^{+}$and $\mathrm{CD} 4^{+}$subsets [38]. After primary infection, EBV establishes a site of latency within the salivary gland epithelial cells and in bone marrow stem cells [5]. In the bone marrow, EBV is probably spread from infected to uninfected cells via the complement CR2 receptor. The specific receptors on salivary gland epithelial cells are not known, but may be similar to complement $\mathrm{CR} 2$ receptors. 
EBV periodically reactivates in the salivary gland (probably several times per year) in asymptomatic normal individuals and the EBV in their saliva becomes the vehicle for infection of the previously uninfected. It is possible to identify specific EBV strains based on specific polymorphisms in their DNA, such as the length of the glycine-alanine repeat in EBNA-1 (BKRF-1) region. Despite the wide number of EBV strains present in the community at a single time, each individual stably carries only a single isolate for lifetime. This implies that immune response to initial infection prevents subsequent EBV infections, even though the immune responses are inadequate to eliminate sites of latency [5]. In patients with immune dysregulation due to underlying desease or to immune-suppressive medications, EBV is reactivated to a greater extent as evidenced by increased levels of virus in saliva, tears and blood.

\section{Potential role of EBV in SS}

Since primary EBV infection induces strong $T$ cell immune responses against EBV and this virus is chronically present in normal salivary glands, it is surprising that all normal salivary glands do not have intense lymphocytic infiltrates in response to viral antigens. Any theories of pathogenesis of SS involving EBV must explain the absence of lymphoid infiltrates in normal salivary glands and the genetic predisposition for SS (i.e., HLA-DR3, -DQA4, DQB2).

Indirect evidence in support of a role for EBV in pathogenesis of SS includes the following: (a) EBV has normal site of latency in salivary and lacrimal glands; thus, the virus is known to be present (in very low amounts) at the target organs of immune response prior to the devolpment of SS; (b) EBV DNA can be found in the saliva and salivary gland biopsies of SS patients in amounts greater than found in normals or in patients with other autoimmune diseases [34]. The use of immunohistological methods for detection of viral proteins [14] and in situ hybridization for viral DNA [27] indicated that EBV is predominantly in the epithelial cells rather than the lymphocytes; (c) in some SS patients, seroconversion for EBV has been observed in association with development of SS [19, 41]; (d) EBV RNA can be associated with the autoantigens SS-A and SS-B [24]; (e) EBV stimulation of B-cells can give rise to rheumatoid factor [8] with the same crossreactive idiotype that is present in SS patients [13]; and (f) there are increased antibody titers against specific synthetic peptides encoded by the glycine-alanine repeat region of EBNA-1 [33] and against early antigen EA-D [14].

EBV may contribute to pathogenesis of SS in several ways. First, EBV-infected cells in the target organ (i.e., salivary and lacrimal gland) may serve as a direct target for attack by immune $T$ cells. The immune damage to infected cells would lead to a decreased secretory ability and contribute to the symptoms of dryness. Although the amount of EBV in the SS salivary gland is 100 - to 1000 -fold greater than that in the normal salivary gland, only a small proportion of the salivary gland epithelial cells probably contain EBV DNA and, thus, additional mechanisms must be considered. Second, immune responses against EBV proteins may lead to crossreactions against cellular proteins and tissue destruction may occur via mechanisms 
of "molecular mimicry." In particular, molecules such as "heat-shock proteins" have a similar peptide sequence to certain EBV-encoded proteins [39] and may provide targets for autoimmune attack. Thus, EBV-encoded or EBV-like proteins are present in the salivary gland and can serve as a stimulus to $\mathrm{T}$ helper cells. Under normal situations, $\mathrm{T}$ suppressor cells and other suppressive mechanisms would be generated to "turn off"' these immune responses. It is likely that such suppressor feed back loops are deficient in the SS patient, although the paucity of knowledge about such suppressor circuits makes it difficult to experimentally test this hypothesis at the present time.

A model for the role of EBV in SS is shown schematically in Fig. 3. In normal salivary glands (panel A), periodic reactivation of EBV occurs in glandular epithelial cells but the virus is shed directly into the ducts along with saliva and is eliminated without stimulating immune respones [42]. In salivary glands of SS patients, the epithelial cells express the cell surface HLA-DR antigen. This is in contrast to normal salivary glands that lack this antigen $[12,25]$. The induction of HLA-D molecules allows EBV-encoded antigens (or other autoantigens) to be expressed on the cell surface in a conformation that can stimulate $\mathrm{CD}^{+} \mathrm{T}$ cells. The resulting release of cytokines from lymphocytes and epithelial cells perpetuates the production of HLA-DR molecules, activation of T cells and B cells, and transformation of small blood vessels into high endothelial venules [12] that attract further lymphocytes into the gland. Antibodies and cytokines produced within the gland enter the blood stream, where they are measured as autoantibodies and as the acute-phase reactants that they induce. Due to the inability of the immune responses to eradicate EBV at its site of latency and reactivation, this cascade of inflammation continues until the gland is destroyed and clinical symptomes of dryness are detected.

In the proposed model, the critical difference between SS patients and normal individuals is the expression of specific HLA-D-encoded genes (i.e.; HLA-DR3, -DQA4, -DQB2) on the salivary gland epithelial cells. This may represent the structural basis for genetic predisposition to SS. As a result, SS patients may have: (a) an excessive $T$ helper cell response to certain antigens (either exogenous viral antigens or endogenous autoantigens); and/or (b) deficient generation of specific "suppressor" $\mathrm{T}$ cells that function to down-regulate the immune responses to specific antigens. For simplicity, the relevant histocompatibility antigen will be referred to as "HLA-DR3"; however, it is difficult to determine whether the most important gene products are encoded by the HLA-DR, HLA-DQ, or other closely linked genes on this region of human chromosome 6.

There is evidence that abnormalitis in immune response are more common in individuals with HLA-DR3. For example, HLA-DR3 is associated with other autoimmune disorders such as thyroiditis, diabetes and SLE. These patients make high titer antibodies against autoantigens and generally develop hypergammaglobulinemia as part of their systemic autoimmune response. In spite of this immune "hyper-responsiveness" associated with HLA-DR3, it is surprising that these patients have deficient responses in certain clinical situations in which the infectious agent is known. For example, hepatitis $\mathbf{B}$ vaccination is now widely used and certain proportion of the "normal" recipients will be nonresponders despite re-vaccination. Most of the non-responders turn out to be HLA-DR3. Also, 


\title{
A Normal salivary gland
}

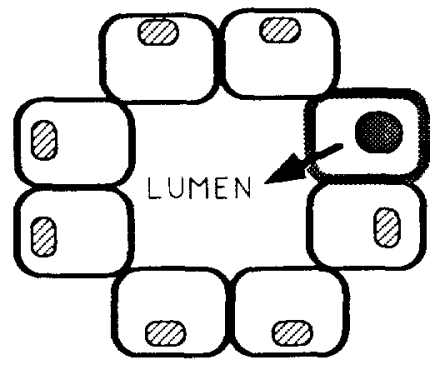

\author{
Salivary gland epithelial cell \\ 1. Viral latency is maintained \\ in a small number of cells, \\ 2. Upon reactivation, \\ EBV shed directly into lumen \\ 3. No induction of HLA-DR antigens \\ 4. No release of factors that induce \\ high endothelial venules
}

\section{B Sjögren's salivary gland}

\section{EBV shed into \\ lumen}

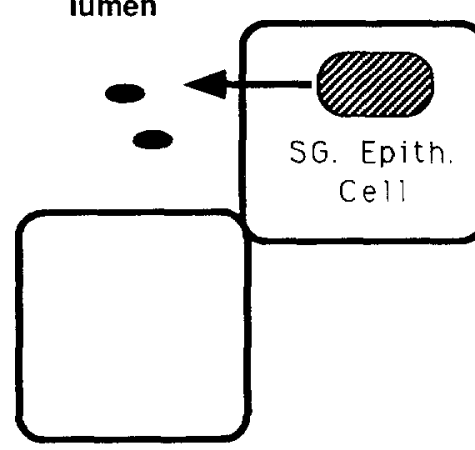

2. HLA-DR induced on Epithelial cell
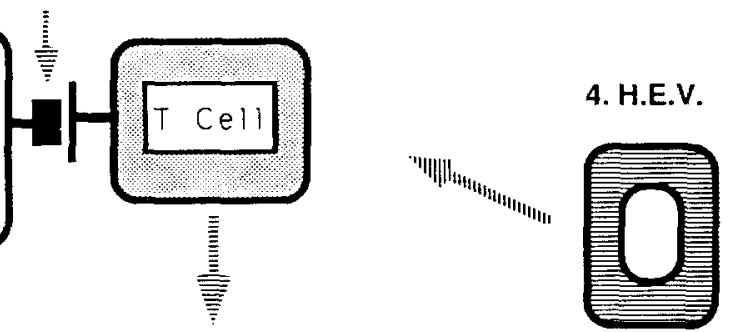

3. Release of Cytokines that:

a) induce further HLA-DR

b) induce high endothelial venules (HEV)

c) activate $T$ cells and $B$ cells

Fig. 3A, B. Schematic model of SS. In A, normal salivary glands may periodically release EpsteinBarr virus (EBV) directly into the duct lumen. Due to the absence of HLA-DR expression, the viral antigens are not recognized by $\mathrm{CD}^{+} \mathrm{T}$ cells. This results in a "privileged" microenvironment where EBV latency and periodic reactivation can occur. In B, salivary gland epithelial (SG. Epith.) cells in SS patients express high levels of HLA-DR molecules. This allows $\mathrm{CD} 4^{+} \mathrm{T}$ cells to recognize viral antigens and release cytokines that further perpetuate the immune response by inducing HLA-DR on other epithelial cells, inducing high endothelial venule that attract further $T$ cells, and activation of $T$ cells and $B$ cells that enter the gland. Despite these immune responses, the EBV can not be eradicated at its site of latency. This self perpetuating immune reaction is not adequately downregulated and the eventual gland destruction leads to clinical symptoms of dryness

infection with leprosy (Mycobacterium leprae) can lead to the tuberculoid or lepromatoid forms of the disease. Individuals with HLA-DR3 have a much higher frequency of the lepromatoid leprosy. Van Eden et al. [39] has shown that HLA$\mathrm{DR}^{+}$lepers have an adequate ability to generate $\mathrm{T}$ helper responses to mycobacterial $65-\mathrm{kDa}$ heat-shock protein and a decreased ability to generate $\mathrm{T}$ suppressor clones to this mycobacterial antigens. As a result, the $T$ helper responses continue to expand without normal regulation by the immune network. An analogous model can be proposed for the immune response to EBV-like antigens in salivary gland of SS patients who are HLA-DR3. 


\section{Development of lymphoma in SS}

The relative risks for $1^{\circ} \mathrm{SS}$ patients of developing lymphoma has been estimated to be approximately 40 -fold higher than age-, sex-matched control subjects by investigators at the NIH [23]. However, Whaley et al. [40] in Glasgow did not find such an increased prevalence. These discrepancies may be attributed to several factors, including the relatively small number of patients reported with lymphoma and ascertainment bias in patient referral patterns. However, these differences also may reflect the difficulty in distinguishing lymphoma from extensive infiltrates due to "benign"' SS or pseudolymphoma [9]. The lymphomas are predominantly non-Hodgkin's B cell (IgM-K) tumors that arise in the salivary gland and cervical lymph nodes. Although the finding of "myoepithelial" islands (i.e., a degenerating tubule surrounded by lymphocytes) is often interpreted as an indication that the tumor is "benign," malignant lymphomas can be found in the same biopsy specimen that contains myoepithelial islands [35]. The distinction between malignant lymphoma and "pseudolymphoma" in SS patients is often quite difficult, even when recombinant DNA methods are utilized [7, 16, 17]. Other forms of non-malignant lymphoid proliferation in $1^{\circ} \mathrm{SS}$ patients include thymoma [2] and angioimmunoblastic lymphadenopathy [30]. In both pseudolymphoma and

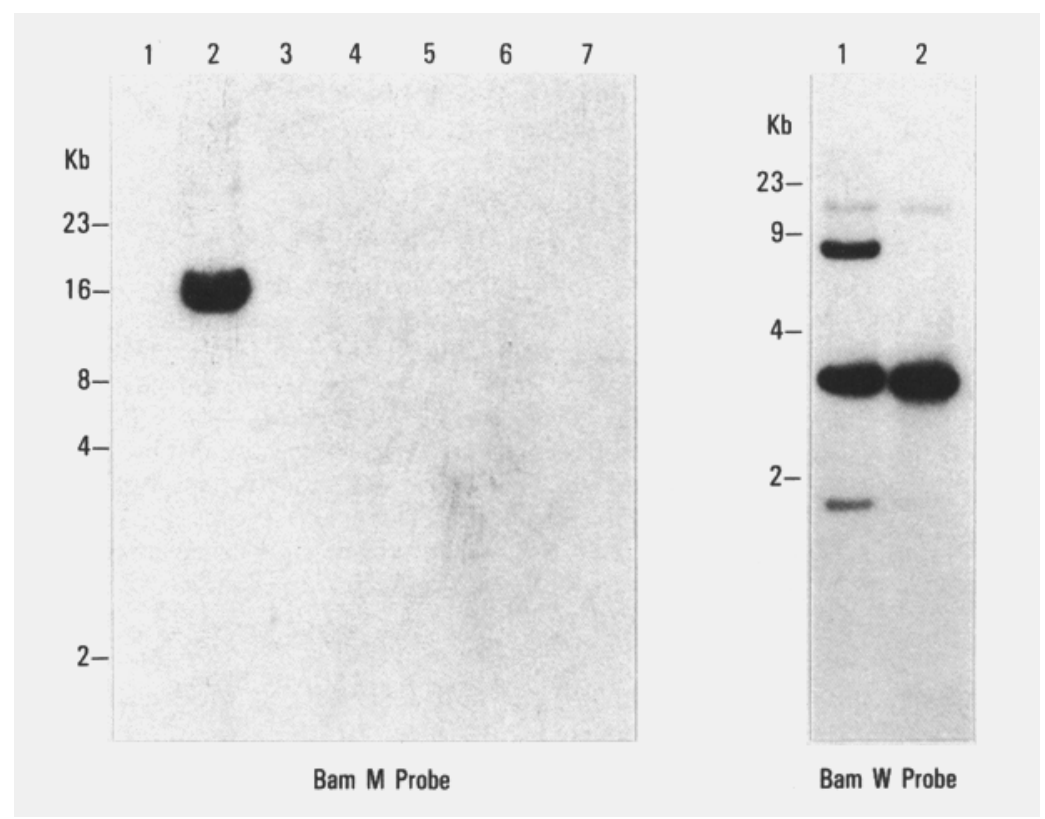

Fig. 4. Detection of EBV DNA in lymph nodes from a SS patient. Left panel. DNA from SS patients who developed non-Hodgkin's B cell lymphoma were digested with Bam $\mathrm{H} 1$ endonuclease and examined with ${ }^{32} \mathrm{P}$-labeled EBV probe BamM. Lanes 1 to 6 correspond to different patient's lymphomas. Right panel. DNA from an EBV-transformed cell line (RPMI 8392) in lane 1 and SS patient lymph node (lane 2) were digested with BamH1 endonuclease and examined with probe EBV BamW 
angioblastic lymphadenopathy, there appears to be a high frequency of progression to frank lymphoma $[23,26]$.

Among $200 \mathrm{SS}$ patients followed for at least 5 years at our institution, nonHodgkin's lymphoma developed in 14 cases. In 9 of these cases, tissue was available for DNA analysis and EBV was detected in the lymphoma tissue from 1 patient using Bam M and BamW probes (lane 2 in Fig. 4, panels A and B, respectively). Due to unusual size of the BamM fragment from this lymphoma after Bam HI enzyme digestion ( $16 \mathrm{~kb}$ instead of the expected $8 \mathrm{~kb}$ ), we digested this lymphoma DNA with two additional restriction enzymes in Fig. 5 (HindIII) in panel A and $E c o$ RI in panel B). We compared the size of the SS lymphoma BamMreactive band (lane 6) to DNA from four different well-established EBV cell lines (lanes 1 to 4 in Fig. 5). The SS lymphoma DNA again showed a different size restriction fragment length polymorphism, indicating that more than a single base pair was altered in the Bam M region of this EBV isolate. This ununusual reactivity was not due to an unusual property of the host cell since lymphocytes from this patient could be transformed by EBV isolate B95-8 to give the expected size (lane 2) and no reactivity was noted with genomic DNA from the same patient (lane 5).

Analysis of terminal repeat (TR) EBV DNA from multiple sites of lymphoma at autopsy of this patient provided interesting results. One tumor site (in the brain) had a two fused TR indicating a "bi-clonal" lymphoma (Fig. 5, lane 5). DNA from a separate site in thoracic lymph node had multiple fused TR (Fig. 5, lane 3 ), as well as lower molecular mass TR (size less than $8 \mathrm{~kb}$ ), indicating replication of the EBV DNA. From these results, we conclude that: (a) most lymphomas in SS patients did not contain EBV DNA; (b) in one SS patient with lymphoma, the

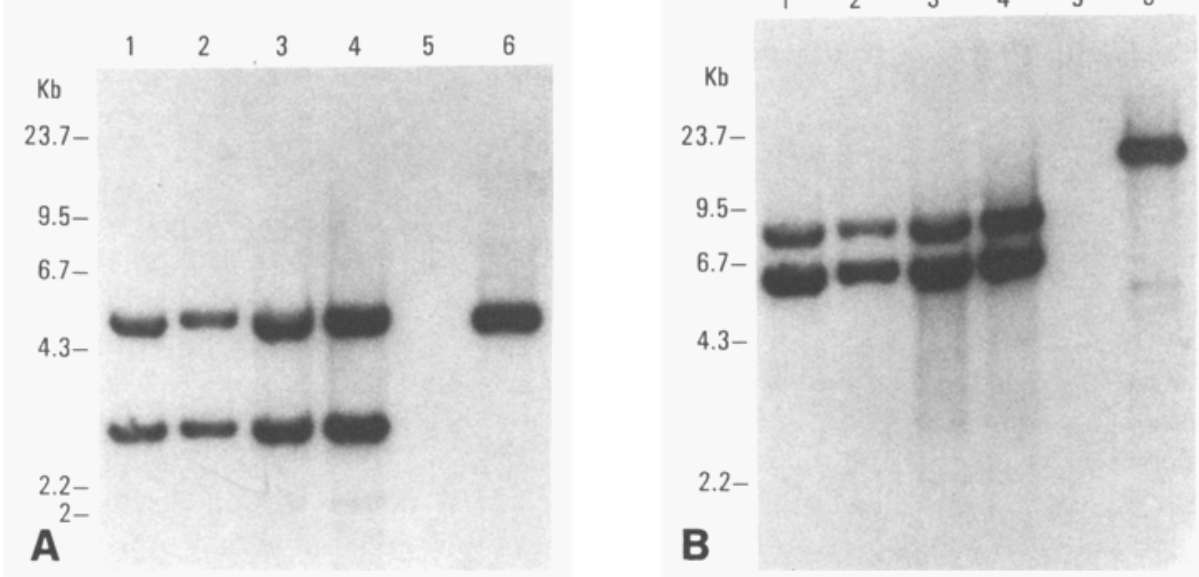

Fig. 5A, B. Unusual restriction fragment size of EBV DNA from SS patient. DNA from cell lines (lanes 1 to 4), from SS patient's lymphoma (lane 6) or from SS patient's granulocytes (lane 5) was digested with Hind III (A) or EcoRI (B). Sources of DNA include: lane 1, EBV-transformed cell line RPMI-8392; lane 2, lymphocytes from SS patient transformed with EBV strain B95-8; lane 3, Wil-2 cell line; lane 4, Daudi cell line; lane 5, granulocyte DNA from SS patient; and lane 6, SS patient's lymphoma DNA 


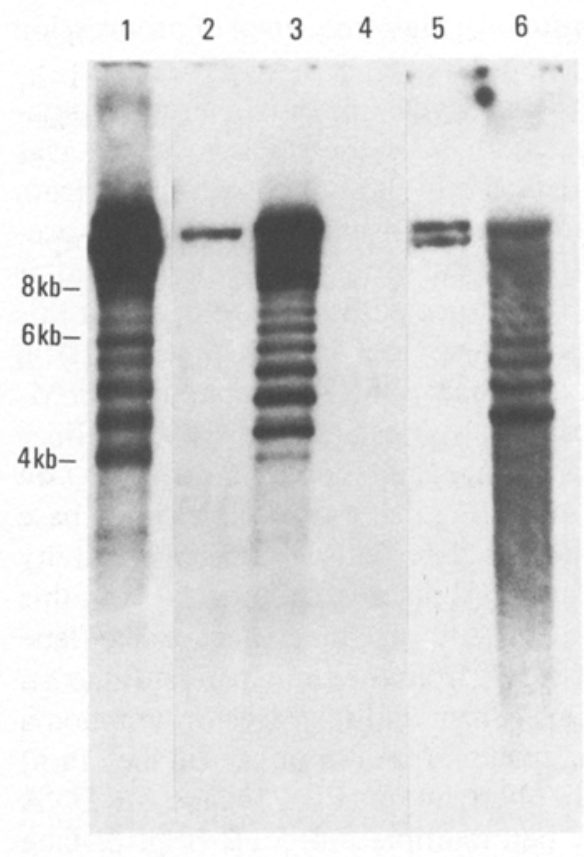

Fig. 6. Different pattern of terminal repeat in SS lymphoma. DNA from different sources was digested with restriction enzyme and reacted with 23P-labeled Xho-1 probe in Southern blot hybridization. Sources of DNA included: lane 1, B95-8 cells; lane 2, Wil-2 cells; lane 3, thoracic lymph node from SS patient with lymphoma; lane 4, granulocyte DNA from SS patient; lane 5 and 6 , brain lymphoma and abdominal lymphoma, respectively, from SS patient

EBV DNA had an unusual size of BamM restriction fragment and multiple forms of TR segment were noted; and (c) more SS lymphomas must be examined before the specific role of EBV can be ascertained.

\section{Potential pitfalls in attributing a role to EBV in the etiology of autoimmune disease}

Although the data might suggest a viral etiology for SS, several lines of evidence indicate the need for caution in assigning EBV a primary etiological role in pathogenesis. First, EBV is a ubiquitous virus and only a few infected individuals get SS; thus EBV infection is not "sufficient" as a cause of disease and can be (at best) a co-factor. Second, primary EBV infection of normals generally occurs prior to age 20 and most autoimmune diseses have a clinical onset over two decades later. This long-lag period makes the assignment of a pathological role for EBV very difficult. Third, although antibody levels against EBV early nuclear antigens (EA-R, EA-D) are elevated [15], the titers appear to rise late in disease and correlate this disease severity; this suggests that EBV reactivation may be a consequence of SS rather than a cause. Fourth, antibody titers to EBV-encoded antigens may be artifactual elevated due to the presence of rheumatoid factor. This autoantibody (IgM anti IgG Fc) will cross-link low-affinity IgG antibodies that would not otherwise react significantly with EBV proteins. Also, antibodies against EBV early antigens (EA-D and EA-R) are generally detected by immunofluorescence methods and ANA present in autoimmune sera may give false positive results. 
To overcome this latter problem in particular, synthetic peptides derived from early antigens have been used in enzyme-linked immunosorbent assays [15]. Fifth, a wide variety of sources for molecular mimicry have been noted based on sequence similarities found in DNA sequence banks. This may partly reflect the fact that herpesvirus DNA sequences constitute a relatively large proportion of DNA sequences in these banks and occurrences of DNA similarities may occur simply by chance.

\section{Conclusions}

EBV is ubiquitous virus that induces strong immune responses among $T$ cells and $B$ cells that persist for life. Despite these immune responses, EBV maintains a life-long site of latency and periodic reactivation in normals, indicating "immunologically privileged" sites of latency and relative inefficiency of the immune system to destroy EBV at these sites of latency. It is likely that this persistent immune stimulation by EBV selects for the expansion of $T$ cells bearing certain cell surface antigen receptors. In this regard, genetic factors such as HLA-DR influence the types of viral antigens that are recognized.

Models of pathogenesis linking EBV and SS can be generated based on the salivary gland serving as a site of EBV latency and reactivation. It is surprising that "normal" salivary glands do not contain lymphoid infiltrates, since immunity to EBV is strongly present in all individuals. The expression of HLA-DR antigens by salivary gland epithelial cells in SS patients (but not in normal salivary glands) may permit the immune recognition of viral antigens by immune $T$ cells. In this model, persistent immune responses to a virus at its site of latency could participate in glandular destruction and eventual clinical symptoms of dryness.

Although indirect evidence has suggested a potential role for EBV in pathogenesis of autoimmune diseases, great caution must be exercised in assigning EBV a primary role in pathogenesis. EBV reactivation and immune cross-reactions may be a consequence of immune dysregulation rather than a cause. Nevertheless, EBV is a very strom immune stimulus that is present for life and therefore, must be considered as a possible agent in the perpetuation of immune dysregulation in genetically predisposed individuals.

Acknowledgements. This is publication number 6881-IMM from the Department of Immunology. This research was supported by research grants AR33983-11 and MO1 RR00833. HK was the Florence Postdoctoral Fellow in Sjögren's Research and was partially supported by a grant from Eiken Chemical, Inc. ML was supported by the Scripps-Stedham Postdoctoral Fellowship and PP by the gift from the Henning and Thornton Charitable Foundations.

\section{References}

1. Adamson TC III, Fox RI, Frisman DM, Howell FV (1983) Immunohistologic analysis of lymphoid infiltrates in primary Sjögren's syndrome using monoclonal antibodies. J Immunol 130: 203

2. Alspaugh M, Whaley K (1982) Sjögren's syndrome. In: Kelly A, Harris A (eds) Textbook of rheumatology, Saunders, Philadelphia, p 971 
3. Ben-Chetrit E, Chan EKL, Sullivan KF, Tan EM (1988) A 52-kDa protein is a novel component of the SS-A-Ro antigenic particle. J Exp Med 167: 1560

4. Block KJ, Buchanan WW, Woho MJ, Bunim JJ (1956) Sjögren's syndrome: a clinical, pathological and serological study of 62 cases. Medicine 44: 187

5. Croen KD (1991) Latency of the human herpesviruses. Annu Rev Med 42: 61

6. Daniels T, Sylvester R S S et al (1974) Tuboreticular structures within the labial salivary glands in Sjögren's syndrome. Arthritis Rheum 17: 593

7. Fishleder A, Tubbs R, Hesse B, Levin H (1987) Immunoglobulin-gene rearrangement in benign lymphoepithelial lesions. N Engl J Med 316: 1118

8. Fong S, Gilbertson TA, Hueniken RJ, Singhal SK, Vaughan JH, Carson DA (1985) IgM rheumatoid factor autoantibody and immunoglobulin-producing precursor cells in the bone marrow of humans. Cell Immunol 95: 157

9. Fox RI, Adamson TC III, Fong S, Robinson CA, Morgan EL, Robb JA, Howell FV (1983) Lymphocyte phenotype and function of pseudolymphomas associated with Sjögren's syndrome. $\mathrm{J}$ Clin Invest $72: 52$

10. Fox RI, Hugli TE, Lanier LL, Morgan EL, Howell F (1985) Salivary gland lymphocytes in primary Sjögren's syndrome lack lymphocyte subsets defined by Leu 7 and Leu 11 antigens. I Immunol 135: 207

11. Fox RI, Theofilopolos AN, Altoman A (1985) Production of interleukin 2 (IL 2) by salivary gland lymphocytes in Sjögren's syndrome. Detection of reactive cells by using antibody directed to synthetic peptides of IL 2. J Immunol 135: 3109

12. Fox RI, Bumol T, Fantozzi R, Bone R, Schreiber R (1986) Expression of histocompatibility antigen HLA-DR by salivary gland epithelial cells in Sjögren's syndrome. Arthritis Rheum 29: 1105

13. Fox RI, Chen PP, Carson DA, Fong S (1986) Expression of a cross-reactive idiotype on rheumatoid factor in patients with Sjögren's syndrome. J Immunol 136: 477

14. Fox RI, Pearson G, Vaughan JH (1986) Detection of Epstein-Barr virus-associated antigens and DNA in salivary gland biopsies from patients with Sjögren's syndrome. J Immunol 137: 3162

15. Fox RI, Scott S, Houghton R, Whalley A, Geltofsky J, Vaughan JH, Smith R (1987) Synthetic peptide derived from the Epstein-Barr virus-encoded early diffuse antigen (EA-D) reactive with human antibodies. J Clin Lab Anal 1: 140

16. Freimark B, Fox RI (1987) Letter. N Engl J Med 317: 1158

17. Freimark B, Fantozzi R, Bone R, Bordin G, Fox R (1989) Detection of clonally expanded salivary gland lymphocytes in Sjögren's syndrome. Arthritis Rheum 32: 859

18. Gaither KK, Fox OW, Yamagata H, Mamula MJ, Reichlin M, Harly JB (1987) Implications of anti-Ro/Sjögren's syndrome A antigen autoantibody in normal sera for autoimmunity. J Clin Invest 79: 842

19. Gaston JS, Rowe M, Bacon P (1990) Sjögren's syndrome after infection by Epstein-Barr virus. J Rheumatol 17: 558

20. Gougerout A (1925) Insuffisance pregressive et atrophie des glandes salivaires et muqueuses de la bouche, des conjonctives (et parfois des muqueuses, nasale, laryngee, vulvarie). "Secheresse", de la bouche, des conjonctives, etc. Bull Soc Fr Dermatol Syphiliar 32: 376

21. Hamilton RG, Harley JB, Bias WB et. al (1988) Two Ro (SS-A) autoantibody responses in systemic lupus erythematous. Arthritis Rheum 31: 446

22. Harley JB, Alexander EL, Bias WB, Fox OF, Provost TT, Richlin M, Yanagata H, Arnett FC (1986) Anti-Ro (SS-A) and Anti-La (SS-B) in patients with Sjögren's syndrome. Arthritis Rheum 29: 196

23. Kassan SS, Thomas TL, Moutsopoulos HM, Hoover R, Kimberly RP, Budman DR, Costa J, Decker JL, Chused TM (1978) Increased risk of lymphoma in sicca syndrome. Ann Intern Med 89: 888

24. Lerner M, Andrews N, Miller J, et al (1982) Two small RNA's encoded by Epstein-Barr virus and complexed with protein are precipitated by antibodies from patients with systemic lupus erythematosus. Proc Natl Acad Sci USA 78: 805

25. Lindahl G, Hedfors E, Kloreskig L, Forsum U (1985) Epithelial HLA-DR expression and T cell subsets in salivary glands in Sjögren's syndrome. Clin Exp Immunol 61: 475

26. Lukes R, Tindle B (1975) Immunoblastic lymphadenopathy. A hyperimmune entity resembling Hodgkin's disease. N Engl J Med 292: I 
27. Mariette X, Gozlan J, Clerc D, Bisson M, Morinet F (1991) Detection of Epstein-Barr virus DNA by in situ hybridization and polymerase chain reaction in salivary gland biopsy specimens from patients with Sjögren's syndrome. Am J Med 90: 286

28. Mikulicz JH (1982) Über eine eigenartige symmetrische Erkrankung der Tränen- und Mundspeicheldrüsen. In: Billroth GT (ed) Beitr Chir Fortschr, Stuttgart, pp 610-620

29. Morgan W, Castleman B (1953) A clinicopathologic study of Mikulicz'a disease. Am J Pathol 29: 471

30. Pierce P, Stern R, Jaffe R, et al (1979) Immunoblastic sarcoma with features of Sjögren's syndrome and SLE in a patient with immunoblastic lymphadenopathy. Arthritis Rheum 22: 911

31. Rader MD, O'Brien C, Liu Y, Harley JB, Reichlin M (1989) Heterogeneity of the Ro/SSA antigen. J Clin Invest 83: 1293

32. Rankow P, Polayes I (1976) Inflammatory conditions of the salivary gland. In: Rankow P (ed) Diseases of the salivary gland. W.B. Saunders, Philadelphia, pp 221-239

33. Rhodes G, Carson DA, Valbracht J, Houghten R, Vaughan JH (1985) Human immune responses to synthetic peptides from the Epstein-Barr nuclear antigen. J Immunol 134: 211

34. Saito I, Servenius B, Compton T, Fox RI (1989) Detection of Epstein-Barr virus DNA by polymerase chain reaction in blood and tissue biopsies from patients with Sjögren's syndrome. J Exp Med 169: 2191

35. Schmid U, Helbron D, Lennert K (1982) Development of malignant lympyhoma in myoepithelial sialadenitis (Sjögren's syndrome). Virchows Arch [A] 395: 11

36. Sestak AL, Harley JB, Yoshida S, Reichlin M (1987) Lupus/Sjögren's autoantibody specificities in sera with paraproteins. J Clin Invest 80: 138

37. Sjögren HS (1933) Zur Kenntnis der keratoconjunctivitis sicca (Keratitis folliformis bei Hypofunktion der Tränendrüsen). Acta Opthalmol (Copenh) 2: 1

38. Thorley-Lawson DA (1988) Basic virological aspects of Epstein-Barr virus infection. Semin Hematol 25: 247

39. van Eden W, Hogervorst J, Hensen E, van der Zee R, van Embden J, Cohen I (1988) Gillis $\mathrm{S}$ (ed) Curr Top Microbiol Immunol, Plenum Press, New York

40. Whaley K, Webb J, McAvoy B, Hughes GR, Lee P, MacSween RN, Buchanan WW (1973) Sjögren's syndrome. II. Clinical associations and immunological phenomena. Q J Med 66: 513

41. Whittingham S, McNeilage J, Mackay IR (1985) Primary Sjögren's syndrome after infectious mononucleosis. Ann Intern Med 102: 490

42. Wolf H, Haus M, Wilmes E (1984) Persistence of Epstein-Barr virus in the parotid gland. J Virol 51: 795

43. Wolin SL, Steitz JA (1984) The Ro small cytoplasmic ribonucleoproteins: identification of the antigenic protein and its binding site on the Ro RNAs. Proc Natl Acad Sci USA 81: 1996 